\title{
Helium Star Donor Channel to Type Ia Supernovae and Their Surviving Companion Stars
}

\author{
Bo Wang ${ }^{1,2}$ and Zhanwen Han $^{1,2}$ \\ ${ }^{1}$ National Astronomical Observatories/Yunnan Observatory, CAS, Kunming, 650011, China \\ (email: wangbo@ynao.ac.cn) \\ ${ }^{2}$ Key Laboratory for the Structure and Evolution of Celestial Objects, CAS, Kunming, \\ 650011, China
}

\begin{abstract}
Employing Eggleton's stellar evolution code and assuming optically thick winds, we systematically studied the He star donor channel of Type Ia supernovae (SNe Ia), in which a carbon-oxygen white dwarf (WD) accretes material from a He main-sequence star or a $\mathrm{He}$ subgiant to increase its mass to the Chandrasekhar mass. We mapped out the initial parameters for producing SNe Ia in the orbital period-secondary mass plane for various WD masses from this channel. Based on a detailed binary population synthesis approach, we find that this channel can produce SNe Ia with short delay times ( 100 Myr) implied by recent observations. We derived many properties of the surviving companions of this channel after SN explosion, which can be tested by future observations. We also find that the surviving companions from the SN explosion scenario have a high spatial velocity $(>400 \mathrm{~km} / \mathrm{s})$, which could be an alternative origin for hypervelocity stars (HVSs), especially for HVSs such as US 708.
\end{abstract}

Keywords. binaries: close — stars: evolution, white dwarfs - supernovae: general

\section{Introduction}

Type Ia Supernovae (SNe Ia) have been applied successfully in determining cosmological parameters, and are also a key part of our understanding of galactic chemical evolution. However, there is still no agreement on the nature of their progenitors. It is generally believed that SNe Ia are thermonuclear explosions of carbon-oxygen white dwarfs (CO WDs) in binaries (Nomoto et al. 1997). Over the past few decades, two families of SN Ia progenitor models have been proposed, i.e., the double-degenerate (DD) and single-degenerate (SD) models. Of these two models, the SD model is widely accepted at present. It is suggested that the DD model, which involves the merger of two CO WDs (Iben \& Tutukov 1984; Webbink 1984), likely leads to an accretion-induced collapse rather than to an SN Ia (Nomoto \& Iben 1985). For the SD model, the companion could be a main-sequence (MS) star or a slightly evolved subgiant star (WD + MS channel), or a red-giant star (WD + RG channel) (Hachisu et al. 1996; Li \& van den Heuvel 1997; Han \& Podsiadlowski 2004, 2006; Chen \& Li 2007; Meng \& Yang 2010; Wang, Li \& Han 2010; Wang \& Han 2010a; Di Stefano et al. 2011). A CO WD may also accrete material from a He star to increase its mass to the Chandrasekhar mass $\left(\mathrm{M}_{\mathrm{Ch}}\right)$; this is known as the He star donor channel. WD + He star systems are believed to originate from intermediate mass binaries, which may explain SNe Ia with short delay times implied by recent observations (Mannucci et al. 2006). The aim of this contribution is to investigate the He star donor channel and to explore the properties of the surviving companions after SN explosion. 


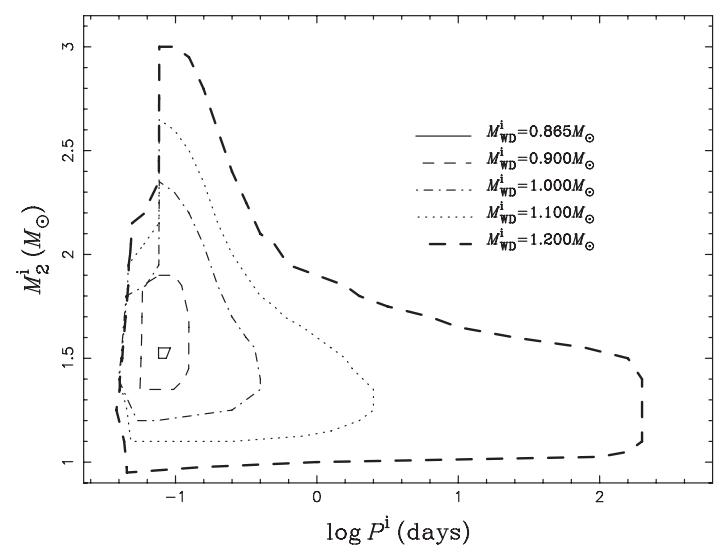

Figure 1. Regions in the orbital period-secondary mass plane for WD binaries (at the onset of the RLOF stage) that produce SNe Ia. (From Wang et al. 2009a)

\section{Binary Evolution Calculations}

We use Eggleton's stellar evolution code (Eggleton 1971) to calculate the evolutions of WD + He star systems. The code has been updated with the latest input physics over the past four decades. In our calculations, He star models are composed by He abundance $Y=0.98$ and metallicity $Z=0.02$. Instead of solving the stellar structure equations of a WD, we use the optically thick wind model (Hachisu et al. 1996) and adopt the prescription of Kato \& Hachisu (2004) for the mass accumulation efficiency of He-shell flashes onto the WD. We have calculated about $2600 \mathrm{WD}+\mathrm{He}$ star systems, and obtained a large, dense model grid (see Wang et al. 2009a). In Figure 1, we show the contours at the onset of Roche lobe overflow (RLOF) for producing SNe Ia in the $\log P^{\mathrm{i}}-M_{2}^{\mathrm{i}}$ plane for various WD masses, where $P^{\mathrm{i}}$ and $M_{2}^{\mathrm{i}}$ are the orbital period and the mass of the He companion star at the onset of RLOF, respectively. If the parameters of a WD + He star system at the onset of the RLOF are located in the contours, an SN Ia is then assumed to be produced. Thus, these contours can be expediently used in binary population synthesis (BPS) studies to investigate the He star donor channel.

\section{Binary Population Synthesis}

To obtain SNe Ia birthrates and delay times of the He star donor channel, we performed a series of Monte Carlo simulations in a BPS study (see Wang et al. 2009b). In the simulation, by using Hurley's rapid binary evolution code (Hurley et al. 2002), we followed the evolution of $4 \times 10^{7}$ sample binaries from star formation to the formation of the $\mathrm{WD}+\mathrm{He}$ star systems based on the SN Ia production regions (Figure 1) and three formation channels (see Wang et al. 2009b). Here, we adopt the standard energy equations to calculate the output of the common envelope phase.

The simulations give Galactic SN Ia birthrate of $\sim 0.3 \times 10^{-3} \mathrm{yr}^{-1}$ by adopting $Z=$ 0.02 and $\mathrm{SFR}=5 M_{\odot} \mathrm{yr}^{-1}$, which is lower than that inferred observationally (i.e., $3-4 \times 10^{-3} \mathrm{yr}^{-1}$; Cappellaro and Turatto 1997). This implies that the He star donor channel is only a subclass of SN Ia production, and there may be some other channels or mechanisms also contributing to SNe Ia (see Wang et al. 2010). The simulations also show the evolution of SN Ia birthrates with time for a single starburst with a total mass of $10^{11} M_{\odot}$. We find that that SN Ia explosions occur between $\sim 45 \mathrm{Myr}$ and $\sim 140 \mathrm{Myr}$ 


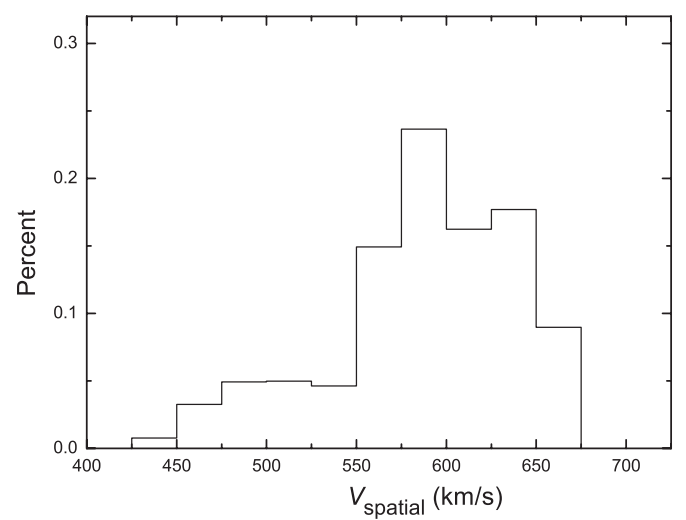

Figure 2. The distribution of the spatial velocity with $\alpha_{\mathrm{ce}} \lambda=0.5$. (From Wang \& Han 2009)

after the starburst, which could explain SNe Ia with short delay times implied by recent observations (Mannucci et al. 2006).

The companion in the SD model would survive in the SN explosion and potentially be identifiable (Wang \& Han 2010b). We obtained many properties of the surviving companions of the He star donor channel at the moment of SN explosion, e.g., the masses, the orbital velocities, the effective temperatures, the surface gravities, the orbital period and the equatorial rotational velocity of companions (see Wang \& Han 2009). These distributions may be helpful for identifying the surviving companions of SNe Ia. The simulation also shows the initial parameters of the primordial binaries and the WD + He star systems that lead to SNe Ia, which may help to search for potential SN Ia progenitors. In Figure 2, we show the current epoch distribution of the spatial velocity for the surviving companions from this channel (see Wang \& Han 2009). We see that the surviving companions have high spatial velocities $(>400 \mathrm{~km} / \mathrm{s})$, which almost exceed the gravitational pull of the Galaxy nearby the Sun. Thus, the surviving companions from the SN explosion scenario could be hypervelocity stars (HVSs), which are stars with velocity so great that they are able to escape the gravitational pull of the Galaxy.

\section{Discussion}

Based on the optically thick wind assumption, Wang \& Han (2010c) recently calculated about 10000 WD + He star systems and obtained SN Ia production regions of the He star donor channel with different metallicities. For a constant star-formation galaxy, they found that SN Ia birthrates increase with metallicity. If a single starburst is assumed, SNe Ia occur systemically earlier and the peak value of the birthrate is larger for a high $Z$. We also note that Liu et al. (2010) recently investigated the effects of rapid differential rotation on the accreting WD, and found that the highest mass of the accreting WD at the moment of SN Ia explosion is $1.81 M_{\odot}$ for the He star donor channel, which may provide a way for the formation of super-Ch mass SNe Ia in the observations.

Massive WD + He star systems are candidates of SNe Ia progenitors. HD 49798/RX J0648.0-4418 provides evidence for the existence of massive WD + He star systems. Based on the data from the XMM-Newton satellite, Mereghetti et al. (2009) recently derived the masses of the two components. The corresponding masses are $1.50 \pm 0.05 M_{\odot}$ for HD 49798 and $1.28 \pm 0.05 M_{\odot}$ for the WD. Based on a detailed binary evolution model, Wang \& Han (2010d) found that the massive WD can increase its mass to $\mathrm{M}_{\mathrm{Ch}}$ during its future evolution. Thus, HD 49798 with its WD companion is a candidate SN Ia 
progenitor. V445 Pup is another massive WD + He star system, in which the mass of the WD is more than $1.35 M_{\odot}$, and the mass of the He star is more than $0.85 M_{\odot}$ (Kato et al. 2008).

US 708 is an extremely He-rich sdO star in the halo of the Galaxy, with a heliocentric radial velocity of $+708 \pm 15 \mathrm{~km} / \mathrm{s}$ (Hirsch et al. 2005). We note that the local velocity relative to the Galactic center may lead to a higher observed velocity for the surviving companions, but this may also lead to a lower observed velocity. Considering the local velocity near the Sun $(\sim 220 \mathrm{~km} / \mathrm{s})$, we find that $\sim 30 \%$ of the surviving companions may be observed to have velocity $V>700 \mathrm{~km} / \mathrm{s}$ for an $\mathrm{SN}$ ejecta velocity of $13,500 \mathrm{~km} / \mathrm{s}$. In addition, the asymmetric explosion of SNe Ia may enhance the velocity of the surviving companions. Thus, a surviving companion in the He star donor channel may have a high velocity like US 708. In future investigations, we will employ the Large sky Area Multi-Object fiber Spectral Telescope (LAMOST) to search for HVSs originating from the surviving companions of SNe Ia.

This work is supported by the Natural Science Foundation of China under grant nos. 10821061, 11033008, 2007CB815406 and 2009CB824800.

\section{References}

Cappellaro, E. \& Turatto, M. 1997, in Ruiz-Lapuente P., Cannal R., \& Isern J., eds, Thermonuclear Supernovae. Kluwer, Dordrecht, P. 77

Chen, W.-C. \& Li, X.-D. 2007, ApJ, 658, L51

Di Stefano, R., Voss, R., \& Claeys, J. S. W. 2011, ApJ, 738, 1

Eggleton, P. P. 1971, MNRAS, 151, 351

Hachisu, I., Kato, M., \& Nomoto, K. 1996, ApJ, 470, L97

Han, Z. \& Podsiadlowski, Ph. 2004, MNRAS, 350, 1301

Han, Z. \& Podsiadlowski, Ph. 2006, MNRAS, 368, 1095

Hirsch, H. A., Heber, U., O'Toole, S. J., \& Bresolin, F. 2005, A\&\&A, 444, L61

Hurley, J. R., Tout, C. A., \& Pols, O. R. 2002, MNRAS, 329, 897

Iben, I. \& Tutukov, A. V. 1984, ApJS, 54, 335

Kato, M. \& Hachisu, I. 2004, ApJ, 613, L129

Kato, M., Hachisu I., Kiyota S., \& Saio, H. 2008, ApJ, 684, 1366

Li, X.-D. \& van den Heuvel, E. P. J.. 1997, A\&A A, 322, L9

Liu, W.-M., Chen, W.-C., Wang, B., \& Han, Z. 2010, A\& A, 523, A3

Mannucci, F., Della Valle, M., \& Panagia, N. 2006, MNRAS, 370, 773

Meng, X. \& Yang, W. 2010, ApJ, 710, 1310

Mereghetti, S., Tiengo, A., Esposito, P., et al. 2009, Science, 325, 1222

Nomoto, K. \& Iben, I. 1985, ApJ, 297, 531

Nomoto, K., Iwamoto, K., \& Kishimoto, N. 1997, Science, 276, 1378

Wang, B. \& Han, Z. 2009, A\&GA, 508, L27

Wang, B. \& Han, Z. 2010a, RAA (Res. Astron. Astrophys.), 10, 235

Wang, B. \& Han, Z. 2010b, MNRAS, 404, L84

Wang, B. \& Han, Z. 2010c, A\&A, 515, A88

Wang, B. \& Han, Z. 2010d, RAA (Res. Astron. Astrophys.), 10, 681

Wang, B., Li, X.-D., \& Han, Z. 2010, MNRAS, 401, 2729

Wang, B., Liu, Z., Han, Y., et al. 2010, ScChG (Sci. China Ser. G), 53, 586

Wang, B., Meng, X., Chen, X., \& Han, Z. 2009a, MNRAS, 395, 847

Wang, B., Chen, X., Meng, X., \& Han, Z. 2009b, ApJ, 701, 1540

Webbink, R. F. 1984, ApJ, 277, 355 